\title{
INFLUENCIA DE LA ESTABILIZACIÓN POR COPIGMENTACIÓN INTERMOLECULAR SOBRE LA CAPACIDAD ANTIOXIDANTE DE ANTOCIANINAS DEL FRUTO DE Vaccinium floribundum Kunth
}

\author{
INFLUENCE OF STABILIZATION BY INTERMOLECULAR \\ COPIGMENTATION ON THE ANTIOXIDANT CAPACITY OF \\ ANTOCIANINES OF THE FRUIT OF Vaccinium floribundum Kunth
}

Martha Suárez H. ${ }^{1}$, Edison Yazán V. ${ }^{2}$ \& Geovanna Narváez Olmos ${ }^{2}$ Recibido: 28 septiembre 2017 / Aceptado: 11 diciembre 2017

Palabras claves: antocianinas, capacidad antioxidante, copigmentación, DPPH, Vaccinium floribundum.

Keywords: anthocyanins, antioxidant capacity, copigmentation, DPPH, Vaccinium floribundum.

\section{RESUMEN}

Se extrajeron antocianinas del fruto mortiño Vaccinium floribundum Kunth mediante percolación y se copigmentaron con: ácido rosmarínico, ácido ferúlico y una mezcla 1:1 de estos ácidos a concentraciones de 120 y 960 mg/L. Se determinó la capacidad antioxidante del extracto antociánico, los complejos de

1 Universidad Central del Ecuador, Facultad de Ciencias Químicas, Carrera de Química, Quito, Ecuador (masuarez@uce.edu.ec)

2 Universidad Central del Ecuador, Facultad de Ciencias Químicas, Carrera de Química Farmacéutica, Quito, Ecuador 
copigmentación, los copigmentos puros, el ácido ascórbico y la cianidina-3glicósido, mediante el método de inhibición del radical 2,2-difenil-1-picrilhidracilo, DPPH. Con los datos experimentales se obtuvieron mediante regresión lineal curvas $\Delta$ Absorbancia versus Concentración. Se utilizó como parámetro de comparación las pendientes de las rectas y se estableció la relación entre la respuesta del estándar de ácido ascórbico y las respuestas obtenidas para cada uno de los compuestos estudiados. Se calculó la relación entre la pendiente del estándar y las pendientes de los compuestos analizados que se expresó como incremento o decremento de la capacidad antioxidante. La expresión química de los resultados, se obtuvo mediante el inverso de la relación entre la pendiente del estándar y de las muestras; a estos valores se asignaron las unidades de medición: Capacidad Antioxidante Equivalente al Ácido Ascórbico, CAEA. Los resultados indicaron que la capacidad antioxidante de los complejos de copigmentación crece en función del tipo y la concentración de copigmento utilizado y que la capacidad antioxidante de los extractos antociánicos es comparativamente menor que la de los complejos de copigmentación. Los copigmentos con ácido rosmarínico y ácido ferúlico a 960 mg/L tienen un mayor incremento en la capacidad antioxidante; el ácido rosmarínico es el que genera incrementos mayores. La copigmentación intermolecular de los extractos antociánicos potencia la capacidad antioxidante de los extractos puros, este efecto es más evidente cuando se utilizan altas concentraciones de copigmentos.

\section{ABSTRACT}

Anthocyanins from mortiño (Vaccinium floribundum Kunth) were extracted by percolation, and stabilized by intermolecular copigmentation with rosmarinic acid, ferulic acid and a mixture 1:1 of these phenolic acids at concentrations of 120 and $960 \mathrm{mg} / \mathrm{L}$. The antioxidant capacity of the non-copigmented anthocyanin extract, copigmented extracts, pure copigments, ascorbic acid and cyanidin-3-glycoside was determined by the DPPH method. Curves $\triangle$ Absorbance vs Concentration were obtained by linear regression with experimental data. The slopes of the curves were used as comparison parameter. A relation was established between the slope of the ascorbic acid standard and slopes of the antioxidant compounds studied. The percentage ratio between the slope of the 
standard and the slope of antioxidants analyzed was calculated as increase or decrease antioxidant capacity. The chemical expression of the results was obtained by an inverse relation between the slope of the standard and samples. These results were named as: Antioxidant Capacity Equivalent to Ascorbic Acid, CAEA (acronym in Spanish). The results indicated that the antioxidant capacity of non-copigmented extracts is lower than from stabilized extracts. Copigmented extracts with rosmarinic acid have a higher antioxidant capacity than copigmented extracts with ferulic acid. The anthocyanic extracts stabilized with mixture 1:1 have different behavior. The antioxidant capacity increases are caused by synergistic effect of the copigments.

\section{INTRODUCCIÓN}

Los índices de mortalidad debidos a las enfermedades crónicas no transmisibles (ECNT), entre las que se incluyen enfermedades cardiovasculares, respiratorias, diabetes y cáncer, han ido en aumento en los últimos años a nivel mundial. La Organización Mundial de la Salud (OMS) estima que para el 2020, el $75 \%$ de las muertes en el mundo serán atribuibles a este tipo de enfermedades (Ferrante et al., 2011). El consumo de productos ricos en antioxidantes está asociado a la prevención y protección contra las ECNT.

Debido a su abundancia y excelentes propiedades colorantes y antioxidantes, las antocianinas son una de las mejores alternativas naturales para combatir estas afecciones. Estos pigmentos naturales tienen propiedades farmacológicas y terapéuticas, vinculadas con su capacidad antioxidante contra el peróxido de hidrógeno y otras especies reactivas de oxígeno, EROS. Así también, a las antocianinas se les atribuye actividad antitumoral y anticancerígena, especialmente a la cianidina-3-glicósido, la más abundante en la naturaleza(Garzón, 2008) . Las antocianinas actúan como antioxidantes, donando hidrógenos y electrones, o bien captando los electrones desapareados de los radicales libres y deslocalizándolos en su estructura aromática (Kuskosky et al., 2004). 
Se han reportado investigaciones sobre las propiedades funcionales de las antocianinas (Gaviria et al., 2009; Kuskosky et al., 2004). En estos documentos, se analizan el contenido y la capacidad antioxidante de antocianinas puras y extractos antociánicos de Vaccinium meridionale. Las investigaciones determinan el alto potencial que tienen estos compuestos como colorantes funcionales.

Las antocianinas son inestables al $\mathrm{pH}$, disolventes, oxígeno, luz, enzimas y otras sustancias. De la misma manera su procesamiento, almacenaje y transporte generan inconvenientes, ya que se degradan por oxidación o reacciones fotoquímicas, por lo tanto, es indispensable estabilizarlas. Una de las técnicas de estabilización más importante es la copigmentación intermolecular, donde se aprovechan las características estructurales de la antocianina y de los copigmentos -ácidos fenólicospara que por medio de interacciones hidrofóbicas, formen complejos estables (Rein, 2005). Debido a que las antocianinas tienen una forma casi plana con electrones $\pi$ deslocalizados, al igual que los copigmentos, hacen que la formación de comple- jos sea uno de los más eficaces medios para proteger el núcleo flavilo rojo (figura $1 \mathrm{~A}$ ), del ataque nucleofílico del agua que es una de las causas para la inestabilidad de los compuestos antociánicos. La formación de puentes de hidrógeno entre el grupo carbonilo de la base quinoidal de las antocianinas con el copigmento, también ha sido sugerida como explicación para la formación del complejo antocianina-copigmento, tal como presenta la figura 1B (Rein, 2005).

A pesar que la copigmentación intermolecular se da por fenómenos físicos de interacción, en la estructura de las antocianinas la formación de puentes de hidrógeno depende de los enlaces polares $\mathrm{OH}$ y carbonilo. El grupo $\mathrm{OH}$ es un donador de protones que potencializa la capacidad antioxidante de los compuestos antociánicos, y al involucrarse en la formación de puentes de hidrógeno generados en la copigmentación intermolecular, puede verse afectada esta propiedad en los complejos antocianina-copigmento.

Los frutos de Vaccinium floribundum Kunth se caracterizan por su alto 
contenido de antocianinas y compuestos fenólicos. Se ha reportado que su composición química está conformada predominantemente por quercetina, derivados del ácido hidroxicinámico y cianidina-3-glucosido. Vasco et al. (2009) determinaron que aproximadamente el $67 \%$ de los compuestos fenólicos totales corresponden a antocianinas (345 mg cianidina/100 g fruto fresco), de las cuales aproximadamente el $89 \%$ son derivados de la cianidina (cianidina galactósido, cianidina glucósido, cianidina arabinósido). Adicio nalmente, aunque en menor proporción, identificaron delfinidina y dos de sus derivados: delfinidina galactósido y delfinidina arabinósido, así como también, cianidina y delfinidina como agliconas.
En relación con los derivados del ácido hidroxicinámico, los mismos autores determinaron que los frutos de mortiño contienen principalmente ácido clorogénico ( 17 mg/100 g fruto fresco) y derivados del ácido cafeico ( 15 mg/100 g fruto fresco). Se encontraron adicionalmente glicósidos de la quercetina (quercetin-3-oglucósido, quercetin-3-o-xilósido, quercetin-3-o-arabonósido y quercetin-3-o-ramnósido). Entre otros compuestos, se estableció también la presencia de derivados del ácido hidroxibenzoico (derivados del ácido vanílico, gálico y p-hidroxibenzoico), flavan-3-oles y proantocianidinas en menor concentración.

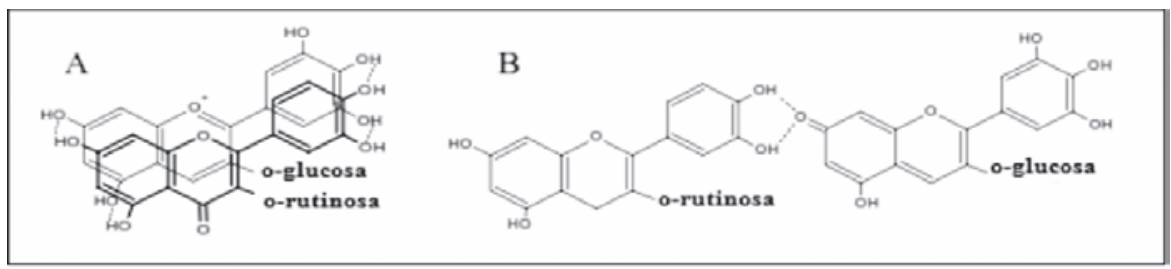

Figura 1. Formación de complejos antocianina-copigmento mediante copigmentación intermolecular. A) Complejo en forma de "sándwich". B) Complejo sugerido mediante enlaces hidrógeno. Fuente: (Rein, 2005) 


\section{MATERIALES Y MÉTODOS}

\section{Cuantificación de cianidina-3-glicó- sido}

Utilizando el fruto de Vaccinium floribundum Kunth colectado en los páramos del cantón Sigchos $0^{\circ} 42^{\prime} 00^{\prime \prime}$ sur $78^{\circ} 53^{\prime} 00^{\prime \prime}$ oeste de la provincia de Cotopaxi, se preparó un extracto etanólico total de acuerdo con la metodología estandarizada (Suárez \& Narváez, 2016). La identificación y cuantificación de la cianidina-3-glicósido en el extracto antociánico se realizó mediante Cromatografía de Capa Fina de Alta Resolución (HPT LC), en un cromatógrafo CAMAG, se utilizó como patrón cianidina-3-glicósido Sigma- Aldrich, CAS 7084-24-4 de acuerdo al método estandarizado (Suárez \& Narváez, 2016), con las condiciones experimentales definidas en la Tabla 1.

\section{Copigmentación intermolecular}

A partir del extracto total, se copigmentaron antocianinas con: ácido ferúlico, ácido rosmarínico y una mezcla 1:1 (v:v) de estos ácidos, a las concentraciones de 120 y 960 mg/L siguiendo la metodología establecida
(Suárez \& Narváez, 2016). El ácido trans-ferúlico y el ácido rosmarínico utilizados corresponden a estándares secundarios Sigma- Aldrich CAS 53798-4 y 20283-92-5, respectivamente.

\section{Determinación de la capacidad an- tioxidante}

La capacidad antioxidante se determinó en un espectrofotómetro UVVIS Varian Bio Cary 50 a 515 nm, mediante la medición de la disminución de absorbancia generada en la disolución del radical 2,2-difenil-1picrilhidracilo (DPPH), cuando reacciona con un compuesto inhibidor de radicales libres. Se utilizó estándar analítico DPPH Sigma-Aldrich, CAS 1898-66-4. Los valores obtenidos para: antocianinas, copigmentos puros y antocianinas copigmentadas se relacionaron con la absorbancia de ácido ascórbico estándar, Sigma - Aldrich CAS 50-81-7 utilizado como referencia (Eiro-Maarit \& Heinonen, 2002; Kim, Lee \& Lee, 2002). Con los datos experimentales se obtuvieron curvas $\triangle$ Absorbancia versus Concentración (Kim et al., 2002), con las cuales se estableció la relación entre 
la respuesta del estándar y la respuesta obtenida para cada uno de los compuestos estudiados. Se utilizó como parámetro de comparación las pendientes de las curvas de acuerdo con el criterio de "comparación directa o nula" (Skoog et al., 2015).

Tabla 1. Condiciones experimentales del desarrollo cromatográfico por HPTLC

\begin{tabular}{|c|c|c|}
\hline Parámetros & & Condiciones de desarrollo \\
\hline Presecado & Tiempo & 30,0 segundos \\
\hline \multirow{3}{*}{$\begin{array}{l}\text { Control } \\
\text { humedad }\end{array}$} & Tiempo & $10,0 \mathrm{~min}$ \\
\hline & Solvente & cloruro de magnesio \\
\hline & Volumen de llenado & $800 \mathrm{~mL}$ \\
\hline \multirow{4}{*}{ Saturación } & Almohadilla de saturación & Sí \\
\hline & Tiempo & $20,0 \mathrm{~min}$ \\
\hline & Volumen de fase móvil & $25 \mathrm{~mL}$ \\
\hline & Solvente & $\begin{array}{l}\text { n-butanol, ácido fórmico, agua } \\
4: 1: 1,5(\mathrm{v} / \mathrm{v} / \mathrm{v})\end{array}$ \\
\hline \multirow{4}{*}{ Desarrollo } & Preacondicionamiento de placa & $10,0 \mathrm{~min}$ \\
\hline & Volumen de fase móvil & $10 \mathrm{~mL}$ \\
\hline & Fase móvil & $\begin{array}{l}\text { n-butanol, ácido fórmico, agua } \\
4: 1: 1,5(\mathrm{v} / \mathrm{v} / \mathrm{v})\end{array}$ \\
\hline & Distancia de migración & $78,0 \mathrm{~mm}$ \\
\hline Secado & Tiempo & $15,0 \mathrm{~min}$ \\
\hline
\end{tabular}

\section{RESULTADOS}

Se identificó cianidina-3-glicosido a de variación de 5,49 \% (Zhao et al., un Rf de 0,35 coincidente con el estándar. La cuantificación se realizó mediante la integración por altura de los cromatogramas del estándar de cianidina-3-glicósido. A partir de un ajuste polinomial, con un coeficiente 2014); se definió que la concentración de cianidina-3-glicósido fue 4,68 \pm $1,13 \mathrm{mg} / \mathrm{g}$ de extracto seco de Vaccinium floribundum Kunth. Se obtuvieron valores de $\Delta$ Absorbancia, mediante la diferencia de absorción entre el 
radical 2,2-difenil-1-picrilhidracilo (DPPH),-absorbancia blanco- y la absorbancia de las muestras analizadas (Kim et al., 2002), para valores de concentración entre $0-2,5 \mathrm{mg} / \mathrm{L}$.

Al seguir igual metodología, se obtuvieron los datos para todos los compuestos de estudio. Estos valores se graficaron en curvas de $\Delta$ Absorbancia versus Concentración para cada compuesto, los valores de coeficien- tes de correlación promedio y de las pendientes de las rectas se resumen en la Tabla 2. Para comparar el comportamiento de la capacidad antioxidante de los compuestos de estudio, se tomó como referencia la pendiente de la recta del estándar de ácido ascórbico, donde los datos se interpretan como el cambio en la reducción de la absorbancia del DPPH, cuando la concentración del antioxidante crece en una unidad (Figura 2).

Tabla 2. Coeficiente de correlación y pendiente de las curvas $\Delta$ Absorbancia versus Concentración y relación de pendientes de los compuestos estudiados

\begin{tabular}{lccc}
\hline Compuesto & $\begin{array}{c}\text { Coeficiente de } \\
\text { correlación }\end{array}$ & $\begin{array}{c}\text { Pendiente de } \\
\text { la recta }\end{array}$ & $\begin{array}{c}\text { Relación de } \\
\text { pendientes }\end{array}$ \\
\hline Écido ascórbico & Estándares & & \\
\hline Cianidina-3-glicósido & 0,9956 & 0,0928 & 1,0000 \\
\hline
\end{tabular}

\section{Extracto antocianinas}

\begin{tabular}{lccc}
\hline Extracto de antocianinas & 0,9969 & 0,0014 & 0,0151 \\
\hline \multicolumn{4}{c}{ Copigmentos } \\
\hline Ácido ferúlico & 0,9929 & 0,0592 & 0,6379 \\
Ácido rosmarínico & 0,9745 & 0,1410 & 1,5194 \\
Mezcla $1: 1$ & 0,9994 & 0,1880 & 2,0259 \\
\hline \multicolumn{4}{c}{ Antocianinas copigmentadas } \\
\hline Extracto $10 \mathrm{mg} / \mathrm{mL}$ - Ac. ferúlico $120 \mathrm{mg} / \mathrm{L}$ & 0,9980 \\
Extracto $10 \mathrm{mg} / \mathrm{mL}$ - Ac. ferúlico $960 \mathrm{mg} / \mathrm{L}$ & 0,9875 & 0,0881 & 0,9494 \\
Extracto $10 \mathrm{mg} / \mathrm{mL}$ - Ac. rosmarínico $120 \mathrm{mg} / \mathrm{L}$ & 0,9987 & 0,1728 & 2,6638 \\
Extracto $10 \mathrm{mg} / \mathrm{mL}$ - Ac. rosmarínico $960 \mathrm{mg} / \mathrm{L}$ & 0,9985 & 0,3335 & 0,8621 \\
Extracto $10 \mathrm{mg} / \mathrm{mL}$ - Mezcla $1: 1 \quad 120 \mathrm{mg} / \mathrm{L}$ & 0,9982 & 0,1953 & 1,3707 \\
Extracto $10 \mathrm{mg} / \mathrm{mL}-$ Mezcla 1:1 $960 \mathrm{mg} / \mathrm{L}$ & 0,9947 & 0,2677 & 2,8847 \\
\hline
\end{tabular}




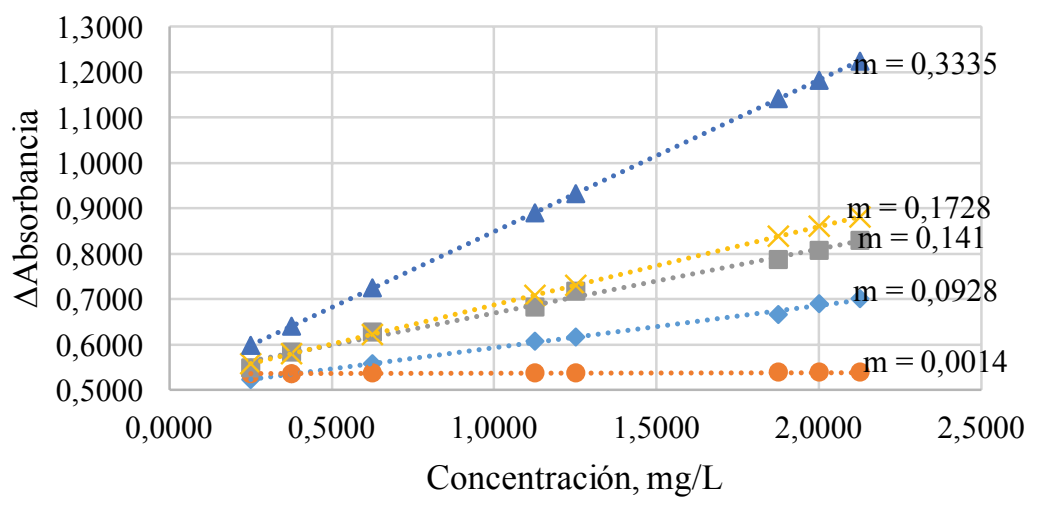

Figura 2 Curvas $\triangle$ Absorbancia versus Concentración del ácido ascórbico $(\diamond)$, extracto de antocianinas (•), ácido rosmarínico ( $($ ), complejo de copigmentación con ácido rosmarínico $120 \mathrm{mg} / \mathrm{L}(\mathrm{x})$, complejo de copigmentación con ácido rosmarínico $960 \mathrm{mg} / \mathrm{L}(\boldsymbol{\Delta})$

Al inverso de la relación entre la pendiente del estándar y de las muestras; se asignó la definición de Capacidad Antioxidante Equivalente a Ácido As- córbico, CAEA. La CAEA es inversamente proporcional a la relación de pendientes descritas en la Tabla 3. 
Tabla 3 Capacidad antioxidante de los compuestos analizados expresados en incremento porcentual y Capacidad Antioxidante Equivalente al Ácido ascórbico (CAEA)

\begin{tabular}{|c|c|c|}
\hline \multirow{2}{*}{ Compuesto } & \multicolumn{2}{|c|}{ Capacidad antioxidante } \\
\hline & \%incremento ${ }^{3}$ & CAEA \\
\hline \multicolumn{3}{|l|}{ Estándares } \\
\hline Ácido ascórbico ${ }^{4}$ & 0 & 0 \\
\hline Cianidina-3-glicósido & $-40,46$ & 1,7380 \\
\hline \multicolumn{3}{|l|}{ Extracto } \\
\hline Extracto de antocianinas & $-98,49$ & 66,23 \\
\hline \multicolumn{3}{|l|}{ Copigmentos } \\
\hline Ácido ferúlico & $-36,21$ & 1,57 \\
\hline Ácido rosmarínico & 51,95 & 0,66 \\
\hline Mezcla 1:1 & 110,45 & 0,48 \\
\hline \multicolumn{3}{|c|}{ Complejos de copigmentación } \\
\hline Extracto 10 mg/mL- Ac. Ferúlico 120 mg/L & $-5,06$ & 1,05 \\
\hline Extracto 10 mg/mL- Ac. Ferúlico 960 mg/L & 166,38 & 0,38 \\
\hline Extracto 10 mg/mL- Ac. Rosmarínico 120 mg/L & 86,21 & 0,54 \\
\hline Extracto 10 mg/mL- Ac. Rosmarínico 960 mg/L & 259,38 & 0,28 \\
\hline Extracto $10 \mathrm{mg} / \mathrm{mL}$ - Mezcla 1:1 $120 \mathrm{mg} / \mathrm{L}$ & 102,59 & 0,49 \\
\hline Extracto $10 \mathrm{mg} / \mathrm{mL}$ - Mezcla 1:1960 mg/L & 188,47 & 0,35 \\
\hline
\end{tabular}

\section{DISCUSIÓN}

A medida que el valor de la pendiente de la recta para cada uno de los compuestos estudiados es mayor que la correspondiente al estándar, el " $\Delta$ Absorbancia" crece, es decir, el compuesto tiene una mayor capacidad antioxidante. Mientras que, cuando el valor de la pendiente es menor al valor de la pendiente del estándar, las curvas tienden a ser paralelas al eje de la concentración debido a que la absorbancia inicial del DPPH no se ve afectada por el escaso efecto antioxidante del compuesto analizado (Figura 2).

3 Los valores negativos corresponden a decremento de la capacidad antioxidante respecto del estándar de ácido ascórbico

4 Se define la capacidad antioxidante del estándar de ácido ascórbico como 100\%. 
Al considerar la relación entre la pendiente de la recta $\Delta$ Absorbancia versus Concentración para el ácido ascórbico y la pendiente de la recta para cada uno de los compuestos analizados (Tabla 2) se puede definir un incremento o decremento de la capacidad antioxidante.

De acuerdo con los criterios analizados, se puede definir que respecto del estándar de ácido ascórbico, los compuestos estudiados tienen los valores de incremento/decremento de capacidad antioxidante y CAEA presentados en la Tabla 3.

Estadísticamente, se definió que al 95 \% de confianza: la concentración, el tipo de copigmento y la combinación de estos dos factores, influyen significativamente sobre la capacidad antioxidante de los complejos de copigmentación intermolecular de las antocianinas del mortiño (Vaccinium floribundum Kunth). La prueba Tukey demuestra que los incrementos de la capacidad antioxidante de los complejos de copigmentación intermolecular de las antocianinas son distintos para cada tratamiento.

El extracto de antocianinas de Vacci- nium floribundum Kunth presentó la capacidad antioxidante comparativamente más baja que los copigmentos (Tabla 3) a pesar que posee cuatro hidrógenos para estabilizar radicales libres, el mismo efecto presenta el ácido rosmarínico. De acuerdo a la estructura presentada en la Figura 3, únicamente los dos hidrógenos de los grupos hidroxilo del anillo F reaccionarían con el radical libre DPPH para que se produzca una estructura estabilizada por hiperconjugación (Kuskosky et.al., 2004).

La capacidad antioxidante de los complejos de copigmentación fue significativamente mayor que la del extracto y los copigmentos puros (Tabla 3), debido a un efecto sinérgico provocado por los copigmentos. Los complejos de copigmentación con ácido rosmarínico, tienen una mayor capacidad antioxidante que los estabilizados con ácido ferúlico, debido a que el ácido rosmarínico tiene cuatro hidrógenos de grupos hidroxilo en sus anillos bencénicos (anillos B y C) disponibles para reaccionar con radicales libres, mientras que el ácido ferúlico solo posee dos (Figura 3). La capacidad antioxidante crece proporcionalmente a la con- 


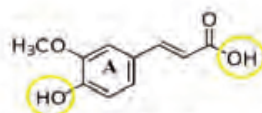

Ácido ferúlico

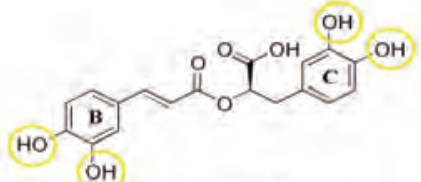

Ácido rosmarínico

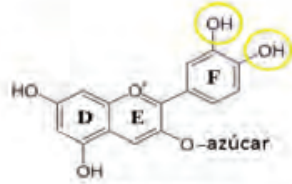

Antocianinas (Estructura general)

Figura 3. Estructuras del ácido ferúlico, ácido rosmarínico y antocianinas.

Fuente: (Arcilla-Lozano, Loarca-Piña, Lecona-Uribe, \& González De Mejía, 2 004; Kuskoski et al., 2004)

centración de las soluciones de los copigmentos usados.

Los complejos de copigmentación con mezcla 1:1 de ácidos ferúlico y rosmarínico a $960 \mathrm{mg} / \mathrm{L}$, tienen una capacidad antioxidante menor que los complejos de copigmentación con ácido rosmarínico, pero mayor que los complejos de copigmentación con ácido ferúlico a la misma concentración. No obstante, a 120 $\mathrm{mg} / \mathrm{L}$, la capacidad antioxidante de los complejos de copigmentación con la mezcla $1: 1$, es mayor que la capacidad antioxidante de los complejos de copigmentación formados con los copigmentos individuales (Tabla 3). Esta variación puede deberse a que el ácido rosmarínico y el ácido ferúlico en solución, además de formar complejos, podrían interaccionar entre sí y con las antocianinas del extracto, formando puentes de hidrógeno y disminuyendo su disponibilidad para estabilizar los radicales libres.

Por lo tanto, la capacidad antioxidante de los complejos de copigmentación con mezcla 1:1 a cualquier concentración tiene un comportamiento diferente a los complejos de copigmentación formados con los copigmentos individuales, donde evidentemente, los complejos de copigmentación con ácido rosmarínico tienen mayor capacidad antioxidante que los formados con ácido ferúlico. 


\section{CONCLUSIÓN}

La copigmentación intermolecular de los extractos antociánicos de Vaccinium floribundum Kunth, genera un incremento en la Capacidad Antioxidante, excepto en el complejo de copigmentación formado con ácido ferúlico a $120 \mathrm{mg} / \mathrm{L}$ en el que se observa un decremento de esta propiedad funcional. El notable incremento de estas propiedades en extractos an- tociánicos potencia las aplicaciones de estos compuestos como colorantes industriales, ya que la copigmentación intermolecular además de tener un efecto positivo sobre la Capacidad Antioxidante estabiliza los colorantes, haciéndolos más resistentes a factores externos como la temperatura y la luminosidad. 


\section{LISTA DE REFERENCIAS}

Arcilla-Lozano, C., Loarca-Piña, G., Lecona-Uribe, S. y González de Mejía, G. (2004). El orégano: propiedades, composición y actividad biológica de sus componentes. Archivos latinoamericanos de nutrición, 54(1), 100-111.

Eiro-Maarit, J. y Heinonen, M. (2002). Anthocyanin color behavior and stability durig storage: Efecct of intermolecular copigmentation. Journal of Agricultural and Food Chemistry, 50(25), 7461-7466.

Ferrante, D., Linetzky, B., Konfino, A., King, A., Virgolini, M. y Laspiur, S. (2011). Encuesta nacional de factores de riesgo 2009: Evolución de la epidemia de enfermedades crónicas no transmsibles en Argentina. Estudio de corte.

Garzón, G. (2008). Las antocianinas como colorantes naturales y compuestos bioactivos. Acta biológica colombiana, 13(3), 27-36.

Gaviria, C., Ochoa, C., Sánchez, N., Medina, C., Lobo, M., Galeano, P., ...y Rojano, B. (2009). Actividad antioxidante e inhibición de la peroxidación lipídica de extractos de frutos de mortiño (Vaccinium meridionale SW). Boletín Latinoamericano y del Caribe de plantas medicinales y aromáticas, 8(6), 519-528.

Kim, D., Lee, H. y Lee, C. (2002). Vitamin C equivalent antioxidant capacity (VCEAC) of phenolic phytochemicals. Journal of Agricultural and Food Chemistry, 50(13), 3713-3717.

Kuskosky, E., Asuero, A., García-Parrilla, M., Troncoso, A. y Fett, R. (2004). Actividad antioxidante de pigmentos antociánicos. Ciencia e Tecnología de Alimentos, 24(4), 691-693.

Rein, M. (2005). Copigmentation reactions and color stability of berry anthociyanins. Helsinky: Universidad Helsinki.

Skoog, D., West, D., Holler, J. y Crounch, S. (2015). Fundamentos de Quimica Analitica. México: Cengage Learning. 
Suárez, M. y Narváez, G. (2016). Copigmentación intermolecular de antocianinas glicosiladas. Madrid: Editorial Académica Española.

Vasco, C., Riihinen, K., Ruales. J. y Kamal-Eldin, A. (2009). Chemical Composition and Phenolic Compound Profile of Mortiño (Vaccinium floribundum Kunth). Journal of Agricultural and Food Chemistry, 57(18), 8274-8281.

Zhao, C., Chen, Z., Bai, X., Ding, C., Long, T., Wei, F. y Miao, K. (2014). Structure-activity relationships of anthocyanidin glycosylation. Molecular Diversity, 18(3), 687-700. 
